\title{
Deep vein thrombosis in a child with osteoarticular infection: A case report
}

\author{
D Shrestha, ${ }^{1}$ H Limbu, ${ }^{2}$ N B Thapa, ${ }^{3}$ A K Dhakal ${ }^{4}$ \\ ${ }^{1,4}$ Department of Pediatrics, ${ }^{2}$ Department of Orthopedics, ${ }^{3}$ Department of Radiology, KIST Medical College, \\ Imadol, Nepal.
}

\begin{abstract}
Acute osteoarticular infections are common in children and rarely complicated with deep vein thrombosis (DVT). This complication can cause significant morbidity and may even be fatal. Herein we report a ten year girl with deep vein thrombosis of left femoral vein who presented initially with septic arthritis of left knee and osteomyelitis of left femur. She required prolonged hospital admission and was managed with surgical debridement, antibiotics and anticoagulation.
\end{abstract}

Key words: Deep vein thrombosis, osteoarticular, osteomyelitis, arthritis, anticoagulation.

\section{Case report}

A 10 year girl was admitted in KIST Medical College Teaching Hospital with complaints of painful swelling of left knee and lower thigh for 2 weeks, accompanied by fever for same duration. There was no history of trauma. On examination, she was febrile and hemodynamically stable. Left knee was semiflexed, swollen, and tender with local rise of temperature and painful movements. Lower half of left thigh was enlarged but ipsilateral leg was normal and Homan's sign was negative. Rest systemic examination was within normal limits. Investigations revealed leukocytosis and X-ray of left knee AP \& lateral views revealed unremarkable findings. Decompression of left knee was done and started on Cloxacillin and Gentamicin intravenously. Despite surgical drainage and parenteral antibiotics for 5 days, her thigh swelling progressed to involve the leg and fever remained persistent. Ultrasonography with color Doppler study was done which revealed periosteal elevation in distal femur with

Correspondence: Dr. Devendra Shrestha

E-mail: devendra.shrestha@gmail.com subperiosteal collection in the medial aspect.There was deep vein thrombosis of left femoral vein extending to left external iliac vein. Repeat X-ray of left thigh showed periosteal reaction involving distal metaphysis of left femur. She was re-explored to decompress acute osteomyelitis and about $50 \mathrm{ml}$ of thick pus was drained from left thigh and femur. Though cultures were sterile, because of unsatisfactory clinical response, antibiotics were changed to Vancomycin. On the same day, anticoagulant therapy was also started with Heparin intravenous infusion for 2 weeks. Later oral warfarin was initiated maintaining INR level of 2-2.5 which was planned to continue for 3 months.

Due to financial constraints, we could not perform detailed prothrombotic work. On repeat Doppler study, thrombus size gradually reduced in size and blood flow was seen. Repeated pus cultures did not reveal any microbial growth. She improved gradually and was discharged after 4 weeks. Later radiographs showed chronic osteomyelitic changes which was managed 
D Shrestha et al, Deep vein thrombosis in a child with

conservatively. She was kept on regular follow up for monitoring of Prothrombin time and INR. She was advised to follow up periodically but was lost in follow up.

Although occurrence of DVT in patients with septic arthritis and osteomyelitis is well known, the incidence is very low in pediatric population and the diagnosis is often delayed and which may interfere with the management. Hence this case was reported intending to highlight this uncommon but serious complication while treating pyogenic infections involving bone and joints.

\section{Discussion}

Deep vein thrombosis is uncommon in children and is most often associated with presence of central venous catheters, trauma, postoperative complications, and inherited thrombotic disorders. ${ }^{1}$ At least one of these risk factors can be found in more than $90 \%$ of children with venous thromboembolism. ${ }^{2}$ Various studies have reported association of thrombosis and septic pulmonary emboli with deep musculoskeletal infection, including osteomyelitis and septic arthritis. ${ }^{3,4,5}$

Infection is a known risk factor for the development of DVT because inflammatory mediators contribute to activated coagulation. Inflammation associated with bone infection can lead to localized endothelial damage and release of cytokines resulting in activation of the coagulation cascade, thrombin generation and fibrin deposition. ${ }^{6}$ This is also associated with local edema and external venous compression which further contributes to venous stasis. ${ }^{2}$ In addition, children with musculoskeletal infection especially involving lower extremities or trunk usually remain immobile, which further increases the risk of DVT. ${ }^{7}$
Although causative microorganism for osteomyelitis in this child could not be isolated, it is well known that Staphylococcus aureus is responsible for most of the acute osteomyelitis in children. ${ }^{8}$ Exotoxin released by S. aureus like Alpha-toxins may produce aggregation of platelets and spasm of smooth muscles. Coagulase enzyme specifically interacts with fibrinogen and causes plasma to clot. ${ }^{9}$ Presence of Pontine-Valentine leucocidin (PVL) genes in S. aureus strain also found to be highly associated with DVT in children with musculoskeletal infections. ${ }^{3,4,5,10}$ It is also important to note that not only bone and joint infections but staphylococcal septicemia can also result into development of DVT in children. ${ }^{11}$ These various factors may predispose to the development of DVT adjacent to osteomyelitis caused by $S$. aureus.

Similarly studies showed that DVT occurred at adjacent site of acute osteomyelitis., ${ }^{3,5,9,12}$ and distal part of femur and proximal part of tibia were the most frequent locations of osteomyelitis associated with deep vein thrombosis, ${ }^{9}$ and femoral and popliteal veins were commonly affected. ${ }^{3}$ It was also observed that majority of DVT associated with bony infections occurred in children aged 8-10 years. ${ }^{3,5}$ Deep vein thrombosis was usually diagnosed after 4 to 6 days following diagnosis of osteomyelitis. ${ }^{3,5,13}$

Deep vein thrombosis is clinically important as it is potentially lethal disease which can lead to thromboembolic complications. Thus prompt diagnosis and timely anticoagulant treatment is crucial to reduce its complications.

However, the clinical features of DVT such as edema, pain, shiny overlying skin overlap with features of acute 
osteomyelitis. Hence presence of DVT might be overlooked in patients with acute osteomyelitis, and DVT is diagnosed only incidentally in many of these cases on color Doppler ultrasonography. ${ }^{3,13,14,15}$ There might be delay in the diagnosis of deep vein thrombosis because of similarity in clinical features. ${ }^{12,13}$ In our case also DVT was detected incidentally when imaging was performed to evaluate the osteomyelitis, though she had features of extremity swelling and pain, consistent with deep vein thrombosis. Though phlebography is still considered the reference standard, ultrasonography is the first choice of investigation for the diagnosis of DVT. $^{3}$

Anticoagulation therapy with Heparin and Warfarin remains the mainstay of initial treatment for DVT. Role of thrombolytic therapy in deep vein thrombosis in children is limited and routine use is not recommended. The thrombus may harbor bacteria leading to a persistent inûammatory response, thus require more prolonged course of antibiotic therapy. ${ }^{13}$ These patients had a longer duration of hospitalization, had more admissions to the intensive care unit, and required more surgical procedures than those with osteomyelitis but without deep venous thrombosis. ${ }^{5}$ Our patient also required prolonged hospitalization and repeated surgical interventions. It has also been suggested that presence of DVT acts as source of septic thrombo emboli which causes dissemination of infection. ${ }^{9,13}$ Presence of DVT in children with musculoskeletal infection is associated with higher rate of disseminated infection which may occur in up to one-third of patients with high mortality. ${ }^{13}$

\section{Conclusion}

Hence a high degree of suspicion for DVT should be considered especially in older children with $S$. aureus musculoskeletal infection involving spine, pelvis, or lower extremities along with a moderately elevated Creactive protein level. ${ }^{5}$ Deep vein thrombosis is uncommon, but one of the serious complications occurring especially in older children with staphylococcal musculoskeletal infections, and because of similarity in clinical features between these, clinicians should be alerted to consider this possibility and initiating appropriate investigations and timely management to reduce the morbidity in these children.

\section{References}

1. P.D. Stein, F. Kayali, R.E. Olson. Incidence of venous thromboembolism in infants and children: data from the National Hospital Discharge Survey. J Pediatr 2004;145:563-5.

2. C.H. van Ommen, M. Peters. Venous thrmboembolic disease in childhood. SeminThromboHemostas 2003;29:392-403.

3. B.E. Gonzalez, J. Teruya, D.H. Mahoney, et al. Venous thrombosis associated with staphylococcal osteomyelitis in children. Pediatrics 2006;117:1673-9.

4. H. Yuksel, A.A. Ozguven, I. Akil, et al. Septic pulmonary emboli presenting with deep venous thrombosis secondary to acute osteomyelitis. PediatrInt 2004;46:621-3.

5. S.T. Hollmig, L.A.B. Copley, R.H. Browne, et al. Deep venous thrombosis associated with osteomyelitis in children. J Bone Joint Surg Am 2007;89:1517-23.

6. A. Amaral, S.M. Opal, J.L. Vincent. Coagulation in sepsis.Int Care Med 2004;30:1032-40.

7. F.A. AndersonJr, F.A. Spencer. Risk factors for venous thromboembolism. Circulation 2003;107:I9-6. 
D Shrestha et al, Deep vein thrombosis in a child with

8. J.A. Herring, editor. Tachdjian's Pediatric Orthopedics. 3rd ed. Philadelphia: Saunders; 2002;1841-72.

9. A. Gorenstein, E. Gross, S. Houri, et al. The pivotal role of deep vein thrombophlebitis in the development of acute disseminated staphylococcal disease in children. Pediatrics 2000;106:e87. Available from: URL:http:// www.pediatrics.org/cgi/content/full/106/6/e87

10. G. Martinez-Aguilar, A. Avalos-Mishaan, K. Hulten, et al. Community-acquired, methicillinresistant and methicillin-susceptible Staphylococcus aureus musculoskeletal infections in children. Pediatr Infect Dis $J$ 2004;23:701-6.
11. R. Prasad, O.P. Mishra, P. Pant. Deep vein thrombosis with Staphylococcusaureus septicemia.IndPediatr 2007;44:43-5.

12. A. Gite, R. Trivedi, U.S. Ali. Deep vein thrombosis associated with osteomyelitis. IndPediatr 2008;45:418-9.

13. S.E. Cray, G.R. Buchanan, C.E. Drake, J.M. Journeycake. Venous thrombosis and thromboembolism in children with osteomyelitis.J Pediatr 2006;149:537-41.

14. S. Walsh, F. Phillips. Deep vein thrombosis associated with pediatric musculoskeletal sepsis. J PediatrOrthop 2002;22:329-32.

15. F.L. Horvath, A.E. Brodeur, J.D. Cherry.Deep thrombophlebitis associated with acute osteomyelitis. J Pediatr 1971;79:815-8. 